\title{
Cardiovascular risk factors in adolescents with prediabetes
}

\author{
S. S. Casagrande ${ }^{1}$, A. Menke ${ }^{1}$, B. Linder ${ }^{2}$, S. K. Osganian ${ }^{2}$, and C. C. Cowie ${ }^{2}$ \\ ${ }^{1}$ Social \& Scientific Systems, Inc., Silver Spring and Digestive and Kidney Diseases, Bethesda, \\ MD, USA \\ ${ }^{2}$ National Institute of Diabetes and Digestive and Kidney Diseases, Bethesda, MD, USA
}

\section{Abstract}

Aims-Adolescents with Type 2 diabetes are more likely to have cardiovascular disease (CVD) risk factors but there are few data available among adolescents with prediabetes. We characterized CVD risk factors among adolescents with prediabetes in the USA and compared levels of those risk factors with adolescents with normal glucose.

Methods-The 2005-2014 National Health and Nutrition Examination Survey, a nationally representative cross-sectional survey, included 2843 adolescents aged 12-19 years after excluding those with diabetes. Prediabetes was based on an $\mathrm{HbA}_{1 \mathrm{c}}$, a fasting plasma glucose or a 2-h plasma glucose. We determined cardiometabolic risk factors in adolescents using age-appropriate cut-off points. We calculated odds ratios (OR) and $95 \%$ confidence intervals (CI) of these outcomes associated with having prediabetes compared with normal glucose levels.

Results-The weighted prevalence of prediabetes was $17.4 \%$. After adjustment, prediabetes (vs. normal glucose) was associated with obesity (OR 1.86, 95\% CI 1.35-2.55), low HDL-cholesterol (OR 1.62, 95\% CI 1.08-2.44), high triglycerides (OR 1.61, 95\% CI 1.12-2.30) and elevated liver transaminase (OR 2.09, 95\% CI 1.19-3.67), but not with hypertension (OR 1.77, 95\% CI 0.883.54), elevated total cholesterol (OR 1.30, 95\% CI 0.82-2.06), elevated LDL-cholesterol (OR 1.59, 95\% CI 0.88-2.88) or albuminuria (OR 1.24, 95\% CI 0.76-2.02).

Conclusions-US adolescents with prediabetes are more likely to have obesity, low HDLcholesterol, high triglycerides and elevated liver transaminase than adolescents with normal glucose. Addressing prediabetes in youth is important for the prevention of Type 2 diabetes and long-term comorbidity.

\section{Introduction}

Children and adults with prediabetes have elevated blood glucose levels that are close to but below the cut-off point for diagnosing diabetes, indicating a higher risk of developing diabetes than those with normal glucose levels [1,2]. Among adults, those with prediabetes have a higher risk of cardiovascular disease (CVD) than those with normal glucose levels [3]. However, there are relatively few data available investigating whether adolescents with prediabetes have elevated levels of CVD risk factors [4-6]. In 2005-2014, the prevalence of

Correspondence to: Sarah S. Casagrande. scasagrande@s-3.com.

Competing interests: None declared. 
prediabetes among adolescents 12-19 years of age in the US general population was $17.7 \%$ [7]. It is well established that those with Type 2 diabetes, even adolescents, are more likely to have CVD risk factors such as obesity [8], hypertension [9] and elevated lipids [10,11]. In fact, risk factors for CVD are often present at the time of Type 2 diabetes diagnosis in adolescents [12]. Because Type 2 diabetes generally takes years to develop, it is unclear if CVD risk factors are present during the prediabetes stage among adolescents.

Our objective was to characterize CVD risk factors among adolescents 12-19 years of age with prediabetes in the US general population, and to compare levels of those risk factors with adolescents with normal glucose levels. We investigated CVD risk factors including obesity, hypertension, LDL-cholesterol, HDL-cholesterol, triglycerides, the liver marker alanine aminotransferase (ALT) and albuminuria in our analysis.

\section{Participants and methods}

\section{Study population}

The National Health and Nutrition Examination Survey (NHANES) is a series of 2-year stratified, multistage probability surveys designed to be representative of the civilian, noninstitutionalized US population [13]. We used data from 2005-2014, the years in which all relevant glucose data were available. The response rate among adolescents was $83 \%$ for the interview and $81 \%$ for the examination. We included adolescents 12-19 years of age who participated in an in-home interview and a visit to a mobile examination centre $(n=2911)$. We excluded adolescents with diagnosed and undiagnosed diabetes [based on American Diabetes Association (ADA) criteria] [1] $(n=62)$ and those who were missing glucose variables $(n=6)$. The final sample size for our analyses included 2843 adolescents; 811 with prediabetes and 2032 with normal glucose levels.

Written informed consent was obtained from adolescents aged 18-19 years; among those aged 12-17 years, written assent was obtained and written informed consent was obtained from parents/guardians. All protocols were approved by the National Center for Health Statistics (NCHS) research ethics board.

\section{Data collection}

During an in-home interview, a standardized questionnaire was used to collect information on age, race/ethnicity, sex, household income and smoking status in the past 5 days [13]. Total number of calories consumed and the percentage of calories from saturated fat were obtained from a 24-h recall.

During the examination, a phlebotomist obtained a blood sample from adolescents according to a standardized protocol [13]. $\mathrm{HbA}_{1 \mathrm{c}}$ was measured and the interassay coefficient of variation ranged from $0.4 \%$ to $1.7 \%$. Although different equipment was used over time, calibration of $\mathrm{HbA}_{1 \mathrm{c}}$ is not necessary according to NHANES recommendations [14]. Onehalf of the adolescents were randomly assigned to a morning examination session and had fasting plasma glucose (FPG) measured; the interassay coefficient of variation ranged from $0.8 \%$ to $2.6 \%$. Also in the morning examination session, an oral glucose tolerance test (OGTT) was administered using a 75-g dose of glucose (or a calibrated dose if the 
adolescent weighed $<42.7 \mathrm{~kg}$ ) and a venepuncture $2 \mathrm{~h}( \pm 15 \mathrm{~min})$ later to obtain a 2-h plasma glucose. We calibrated glucose measurements as recommended by the NCHS [15]. Based on ADA criteria, we categorized adolescents as having prediabetes if they did not have diabetes but had an $\mathrm{HbA}_{1 \mathrm{c}}$ of 39-46 mmol/mol (5.7-6.4\%), a FPG of 5.5-7.0 mmol/1 or a 2-h plasma glucose of $7.8-11.1 \mathrm{mmol} / \mathrm{l}[1]$.

Adolescents had their weight measured using a digital scale and height measured using a stadiometer and BMI was calculated as weight in kilograms divided by height in metres squared. Obesity was defined as having a BMI $\geq 95$ th percentile of age- and sex-specific NCHS growth charts for those aged $12-17$ years $\geq 30 \mathrm{~kg} / \mathrm{m}^{2}$ for adolescents aged $18-19$ years [16]. Blood pressure was measured up to three times and averaged with the first measure excluded. Hypertension was defined as current use of antihypertensive medication or an average systolic blood pressure (BP) or diastolic BP $\geq 95$ th percentile of age-, sex- and height-specific blood pressure for adolescents aged 12-17 years or $\geq 140 / 90 \mathrm{mmHg}$ for those aged 18-19 years [17]. Total cholesterol, HDL-cholesterol and triglycerides were measured enzymatically. We defined high total cholesterol as levels $\geq 11.1 \mathrm{mmol} / \mathrm{l}$, low HDL-cholesterol as $<2.3 \mathrm{mmol} / \mathrm{l}$ and high triglycerides as $27.3 \mathrm{mmol} / \mathrm{l}$ [18]. LDLcholesterol was estimated based on the Friedewald equation [19] and high LDL-cholesterol was defined as levels $\geq 7.3 \mathrm{mmol} / \mathrm{l}$ [18]. ALT was measured and elevated ALT was defined as levels above the assay's reference range for adolescents 12-19 years of age (> $37 \mathrm{IU} / \mathrm{l}$ in males and > $30 \mathrm{IU} / \mathrm{l}$ in females) [13]. A casual (or spot) urine sample was collected and albumin and creatinine were measured. We defined albuminuria as an albumin-to-creatinine ratio $\geq 30 \mathrm{mg} / \mathrm{g}$ [20].

\section{Statistical analysis}

We calculated means or percentages of participant characteristics by prediabetes status. We then calculated the percentage with prediabetes overall and stratified by age, race/ethnicity, sex and BMI. We used logistic regression to estimate the odds ratios (OR) and 95\% confidence intervals (CI) of cardiovascular risk factors including obesity, hypertension, high total cholesterol, high LDL-cholesterol, low HDL-cholesterol, high triglycerides, elevated ALT and albuminuria (microalbuminuria in $90 \%$ of these adolescents and macroalbuminuria in $10 \%$ ) associated with having prediabetes. In these logistic regression models, we also used conditional margins to estimate the percentage with each outcome by prediabetes status. Three models were assessed: (1) unadjusted; (2) adjusted for age, race/ethnicity and sex; and (3) additionally adjusted for household income, smoking status, daily calories consumed, percentage of calories from saturated fat, BMI (except when obesity was the outcome) and systolic BP (albuminuria model only). For outcomes significantly associated with prediabetes, we characterized the shape of the association between glucose markers $\left(\mathrm{HbA}_{1 \mathrm{c}}, \mathrm{FPG}, 2\right.$-h plasma glucose) and the outcomes using restricted quadratic splines with knots at the 10th and 50th percentiles of the weighted glucose distribution and a third knot at the prediabetes cut-off point $39 \mathrm{mmol} / \mathrm{mol}(5.7 \%)$ for $\mathrm{HbA}_{1 \mathrm{c}}, 5.5 \mathrm{mmol} / \mathrm{l}$ for FPG and 7.8 $\mathrm{mmol} / \mathrm{l}$ for 2-h plasma glucose; these cut-off points were generally close to the 90th percentile of the weighted distribution. Spline analyses show the relative odds of the outcome compared with a reference point equivalent to an $\mathrm{HbA}_{1 \mathrm{c}}$ of $31 \mathrm{mmol} / \mathrm{mol}(5 \%)$, a FPG of $4.7 \mathrm{mmol} / \mathrm{l}$ or a 2 -h plasma glucose of $5.5 \mathrm{mmol} / \mathrm{l}$. Because the spline analysis shows 
the relative odds throughout the range of glucose values, the reference point is arbitrary; for the current analysis, the reference values were chosen because they were approximately in the middle of the distribution. Spline analyses used adjustment identical to the final adjusted models above.

Appropriate sample weights were used for all analyses so that the sum of the sample weights (MEC, fasting and OGTT weights) was equivalent to the total civilian non-institutionalized US population; weights were used to account for unequal probabilities of selection and nonresponse, and thus provide estimates representative of the non-institutionalized US population. Data were analysed in 2017 using SUDAAN (version 10.0.1; RTI International, Research Triangle Park, NC, USA) to account for the stratified, clustered sample design used by NHANES.

\section{Results}

Participants with prediabetes were less likely to be non-Hispanic white and more likely to be Hispanic (Table 1). They were also less likely to be female and were more likely to be obese. The weighted per cent with prediabetes was $17.4 \%$ of adolescents (Fig. 1; Table A1). Prediabetes was more common in those aged $12-15$ years (19.5\%) than those aged 16-19 years $(15.3 \% ; P=0.01)$. Prediabetes was more common in non-Hispanic Black $(20.1 \% ; P=$ $0.02)$ and Hispanic participants $(22.2 \% ; P=0.002)$ than non-Hispanic white participants $(15.2 \%)$. Males $(21.4 \%)$ were more likely to have prediabetes than females $(13.3 \% ; P<$ $0.001)$ and obese adolescents (24.4\%) were more likely to have prediabetes than those who were not obese $(15.4 \% ; P<0.001)$. There was no difference in the prevalence of prediabetes by income level $(P=0.37)$ or smoking status $(P=0.62)$.

In unadjusted models, prediabetes was statistically significantly associated with obesity (OR 1.78), hypertension (OR 2.36), low HDL-cholesterol (OR 2.04), high triglycerides (OR 1.87) and elevated ALT (OR 2.73), but not elevated total cholesterol, elevated LDL-cholesterol, or albuminuria (Table 2). After multivariable adjustment, prediabetes was statistically significantly associated with obesity (OR 1.86), low HDL-cholesterol (OR 1.62), high triglycerides (OR 1.61) and elevated ALT (OR 2.09), but not hypertension, elevated total cholesterol, elevated LDL-cholesterol or albuminuria. Prediabetes interaction terms with race/ethnicity and with sex were not significant for any of the outcomes (all $P$-interaction $>0.05)$.

For adolescents with normal glucose levels and those with prediabetes, the multivariable adjusted percentage with obesity was $17.7 \%$ and $28.6 \%$, respectively $(P<0.001)$, with low HDL-cholesterol was $7.8 \%$ and $12.1 \%$, respectively $(P=0.02)$, with high triglycerides was $8.9 \%$ and $13.6 \%$, respectively $(P=0.01)$, and with elevated ALT was $2.9 \%$ and $5.9 \%$, respectively $(P=0.01)$ (Table 2$)$.

The multivariable relative odds of obesity, low HDL-cholesterol, high triglyceride levels and elevated ALT associated with $\mathrm{HbA}_{1 \mathrm{c}}$, FPG and 2-h plasma glucose are shown in Fig. 2. For $\mathrm{HbA}_{1 \mathrm{c}}$, the odds of obesity, low HDL-cholesterol and elevated ALT were relatively flat below $31 \mathrm{mmol} / \mathrm{mol}(5 \%)$ and they began to increase at around 31-37 mmol/mol (5.0- 
$5.5 \%$ ), while the odds of high triglycerides decreased with increasing levels of $\mathrm{HbA}_{1 \mathrm{c}}$. For FPG and 2-h plasma glucose, there was variation in the shape of the associations with each outcome, particularly at lower levels, but the odds of all four outcomes increased as FPG and 2-h plasma glucose levels increased, with the increasing odds generally beginning at levels above an FPG of 4.7-5.3 mmol/l and above a 2-h plasma glucose of $4.5 \mathrm{mmol} / \mathrm{l}$.

\section{Discussion}

In this population-based study of 2843 adolescents age $12-19$ years in the US general population, $17.4 \%$ (weighted) had prediabetes. Adolescents with prediabetes were more likely to be obese, and independent of obesity, they were more likely to have low HDLcholesterol, high triglyceride levels and elevated ALT. The relative odds of obesity, low HDL-cholesterol, high triglycerides and elevated ALT generally began to increase at levels below the prediabetes cut-off points for $\mathrm{HbA}_{1 \mathrm{c}}$, FPG and 2-h plasma glucose. Hypertension was significantly more common in those with prediabetes, but not statistically significant after adjustment for obesity, whereas total cholesterol, LDL-cholesterol and albuminuria did not differ among adolescents with and without prediabetes.

The prevalence of prediabetes in US adolescents varies by age, comorbidities and whether prediabetes is defined by the ADA (> $5.6 \mathrm{mmol} / \mathrm{l}$ ) or World Health Organization (WHO; > $6.1 \mathrm{mmol} / \mathrm{l}$ ) fasting glucose criterion. Previous literature has shown that the prevalence in the USA was $2-9 \%$ based on the WHO criteria and $15-47 \%$ based on the ADA criteria [21]. These estimates align with our results; in subgroup analysis we found that the prevalence of prediabetes ranged from $13.3 \%$ among females to $24.4 \%$ among those who were obese. The higher prevalence of prediabetes among males compared with females may be a result of puberty affecting glucose variability differently by sex; glucose homeostasis during puberty is a relatively unstudied mechanism. In addition, we found that adolescents age 12-15 years had a higher prevalence of prediabetes than those age 16-19 years. This finding may be related to glucose homeostasis in normal weight youth during puberty, a relatively unstudied mechanism. In supplemental analysis we found that the prevalence of prediabetes among non-obese youth age 12-15 years was higher compared to non-obese youth age 16-19 years (17.9\% vs. $12.9 \%)$.

The relatively few studies investigating the association between prediabetes in adolescents and cardiovascular risk factors have not been consistent, except for the association with measures of adiposity. A previous study in NHANES 2005-2006 $(n=777)$ found that adolescents with prediabetes were more likely to be obese and to have a composite outcome defined as at least two of the following risk factors: high waist circumference, hypertension, high triglycerides and low HDL-cholesterol [4]. A study in NHANES 1999-2004 $(n=2764)$ found that adolescents with prediabetes had higher levels of triglycerides and non-HDLcholesterol, but no difference in LDL-cholesterol or HDL-cholesterol [5]. In a crosssectional study conducted in Turkey $(n=198)$ and one conducted in China $(n=933)$, children and adolescents with prediabetes had higher levels of adiposity, but not other cardiovascular risk factors such as hypertension or lipids [22,23]. Conversely, in a crosssectional study conducted in Mexico among children 6-13 years of age $(n=1238)$, impaired fasting glucose was not associated with obesity or LDL-cholesterol but was associated with 
higher levels of total cholesterol and higher levels of HDL-cholesterol [24]. Differences in these studies may be due to a focus on different age ranges, the studies being conducted in different countries, the use of different definitions for prediabetes and CVD risk factors and/or adjustment for different covariates. Our study found that prediabetes was not associated with total cholesterol or LDL-cholesterol; however, it was associated with obesity, low HDL-cholesterol and high triglyceride levels. In addition, prediabetes was associated with hypertension in partially adjusted models, but not in models including adjustment for obesity; a stronger association between prediabetes and hypertension may be shown with a lower blood pressure cut-point, which was recently recommended for adults [25]. These findings are similar to findings in adults with prediabetes [26].

We also found an association between prediabetes and elevated ALT. An association with liver damage/disease is consistent with a previous cross-sectional study of children $<18$ years of age with non-alcoholic fatty liver disease that found those with prediabetes were more likely to have progressed to non-alcoholic steatohepatitis [6].

In our study, the prevalence of prediabetes was higher among those 12-15 years of age than among those 16-19 years of age. This difference may be due to random variability or it may be due to more children going through puberty in the younger age group or the use of cut-off points that are not appropriate for this population [26]. There is substantial discordance between the three markers used to diagnose diabetes and prediabetes. For example, one study found that among adolescents with $\mathrm{HbA}_{1 \mathrm{c}}$ in the prediabetes range $(39-46 \mathrm{mmol} / \mathrm{mol}$, $5.7-6.4 \%), 53 \%$ had a 2 -h plasma glucose in the normal range [27]. Furthermore, there is variability over time as children and adolescents age. Among sixth-grade students with an $\mathrm{HbA}_{1 \mathrm{c}}$ in the prediabetes range, $40 \%$ had $\mathrm{HbA}_{1 \mathrm{c}}$ that regressed to the normal range when measured 2 years later and among those with FPG in the prediabetes range $(5.5-7.0 \mathrm{mmol} / \mathrm{l})$, $52 \%$ had FPG that regressed to the normal range 2 years later [28]. In our study, if participants with normal glucose were misclassified as having prediabetes, then it would likely attenuate associations with the cardiovascular risk factors as long as levels of the outcomes did not affect measurement of the glucose markers. In supplemental analysis, we found that the association with obesity, hypertension, LDL-cholesterol and HDL-cholesterol was stronger among those defined with prediabetes based on having any two diagnostic criteria than those defined by only one diagnostic criteria.

The cross-sectional nature of our study is a limitation, as the possibility of reverse causation or that both prediabetes and the CVD risk factors were a result of a common cause prevents us from determining causality in the observed associations in our study. Additionally, there were relatively few cases of hypertension, which limited power when comparing people with and without prediabetes (prevalence of hypertension was $4.3 \%$ vs. $1.8 \%$, respectively). We also had too few cases of chronic kidney disease to include in our study, but we had sufficient power to include albuminuria. We did not have data on pubertal stage and could not account for any effect that puberty may have had on blood glucose or CVD risk factors. Finally, glucose measures are subject to variability, were only available at one time-point and could not be repeated. However, the ADA does not require a second glucose measure to determine prediabetes [29]. 
Despite the limitations, our study maintained several important strengths. The decade-long 2005-2014 NHANES was a large study, allowing us to identify a relatively large number of adolescents with prediabetes and a sufficient number of most of the outcomes in our analysis. In addition, NHANES was designed to be representative of the civilian, noninstitutionalized US general population. The NHANES data were collected using rigorous study protocols that did not meaningfully change over time and included thorough qualitycontrol procedures and the use of technicians trained and certified in data collection.

We found that $17.4 \%$ of our population had prediabetes and this group of adolescents was more likely to be obese and to have additional cardiovascular risk factors independent of obesity including low HDL-cholesterol, high triglyceride levels and elevated ALT. These findings extend the association of Type 2 diabetes and cardiovascular risk factors in adolescents to those with prediabetes. Indeed, we observed increasing odds of these outcomes beginning at levels below the cut-off point for prediabetes, suggesting that insulin resistance may be the underlying pathophysiology for these risk factors. The issue is particularly concerning because prediabetes affects a large proportion of adolescents and the prevalence has been increasing over time [30]. Our findings highlight a pressing need for further research regarding Type 2 diabetes and prediabetes in adolescents and the long-term health consequences of developing these conditions so early in life. In addition, there is a need for physicians to address the cardiovascular risk factors that are prevalent in a large proportion of US adolescents regardless of their glycaemic status because reducing obesity may help reduce the development of Type 2 diabetes and reducing other risk factors may reduce diabetic complications. Given the long life expectancy for adolescents, physicians should consider both lifestyle and pharmacological treatment options for their patients with prediabetes and/or cardiovascular disease risk factors.

\section{Acknowledgments}

Funding sources: This work was supported by a contract (GS10F0381L) from the National Institute of Diabetes and Digestive and Kidney Diseases (NIDDK). The findings and conclusions in this report are those of the authors and do not necessarily represent the official position of the NIDDK.

\section{Appendix}

Table A1

Weighted percent with prediabetes overall and stratified by participant characteristics, 2005-2014 National Health and Nutrition Examination Survey

\begin{tabular}{lcc}
\hline & Percent (95\% CI) & $P$-value \\
\hline & $\boldsymbol{N}=\mathbf{2 8 4 3}$ & \\
Overall & $17.4(15.5-19.3)$ & - \\
Age (years) & & \\
$12-15$ & $19.5(16.9-22.1)$ & Reference \\
$16-19$ & $15.3(12.8-17.7)$ & 0.01 \\
Race/ethnicity & &
\end{tabular}




\begin{tabular}{lcc}
\hline & Percent $\mathbf{( 9 5 \%}$ CI $)$ & P-value \\
\hline & N $=\mathbf{2 8 4 3}$ & \\
Non-Hispanic white & $15.2(12.2-18.3)$ & Reference \\
Non-Hispanic Black & $20.1(17.7-22.6)$ & 0.02 \\
Hispanic & $22.2(19.2-25.2)$ & 0.002 \\
Sex & & \\
Male & $21.4(18.9-23.8)$ & Reference \\
Female & $13.3(10.6-16.0)$ & $<0.001$ \\
Household income (\$) & $19.0(15.4-22.7)$ & Reference \\
$<$ 20 000 & $16.9(14.5-19.3)$ & 0.37 \\
$\geq 20000$ & $18.4(12.6-24.2)$ & Reference \\
Smoking & $16.9(14.9-18.9)$ & 0.62 \\
Yes & & \\
No & $15.4(13.5-17.3)$ & Reference \\
BMI & $24.4(19.5-29.4)$ & $<0.001$ \\
Not obese & \\
Obese &
\end{tabular}

\section{References}

1. American Diabetes Association. Diagnosis and classification of diabetes mellitus. Diabetes Care. 2010; 33(Suppl 1):S62-69. [PubMed: 20042775]

2. Vijayakumar P, Nelson RG, Hanson RL, Knowler WC, Sinha M. HbA $1 \mathrm{c}$ and the prediction of Type 2 diabetes in children and adults. Diabetes Care. 2017; 40:16-21. [PubMed: 27810987]

3. Huang Y, Cai X, Mai W, Li M, Hu Y. Association between prediabetes and risk of cardiovascular disease and all cause mortality: systematic review and meta-analysis. BMJ. 2016; 355:i5953. [PubMed: 27881363]

4. Li C, Ford ES, Zhao G, Mokdad AH. Prevalence of pre-diabetes and its association with clustering of cardiometabolic risk factors and hyperinsulinemia among US adolescents: National Health and Nutrition Examination Survey 2005-2006. Diabetes Care. 2009; 32:342-347. [PubMed: 18957533]

5. Liu J, Joshi D, Sempos CT. Non-high-density-lipoprotein cholesterol and cardiovascular risk factors among adolescents with and without impaired fasting glucose. Appl Physiol Nutr Metab. 2009; 34:136-142. [PubMed: 19370043]

6. Newton KP, Hou J, Crimmins NA, Lavine JE, Barlow SE, Xanthakos SA, et al. Prevalence of prediabetes and Type 2 diabetes in children with nonalcoholic fatty liver disease. JAMA Pediatr. 2016; 170:e161971. [PubMed: 27478956]

7. Menke A, Casagrande S, Cowie CC. Prevalence of diabetes in adolescents aged 12 to 19 years in the United States, 2005-2014. JAMA. 2016; 316:344-345. [PubMed: 27434447]

8. Liu LL, Lawrence JM, Davis C, Liese AD, Pettitt DJ, Pihoker C, et al. Prevalence of overweight and obesity in youth with diabetes in USA: the SEARCH for Diabetes in Youth study. Pediatr Diabetes. 2010; 11:4-11. [PubMed: 19473302]

9. Brar PC. Vascular phenotype of obese adolescents with prediabetes and/or Type 2 diabetes (T2DM): review of the current literature. Diabetes Metab Syndr. 2016; 10:250-256. [PubMed: 27381966]

10. Kershnar AK, Daniels SR, Imperatore G, Palla SL, Petitti DB, Pettitt DJ, et al. Lipid abnormalities are prevalent in youth with type 1 and Type 2 diabetes: the SEARCH for Diabetes in Youth Study. J Pediatr. 2006; 149:314-319. [PubMed: 16939739] 
11. Lipid and inflammatory cardiovascular risk worsens over 3 years in youth with Type 2 diabetes: the TODAY clinical trial. Diabetes Care. 2013; 36:1758-1764. [PubMed: 23704675]

12. Nadeau KJ, Anderson BJ, Berg EG, Chiang JL, Chou H, Copeland KC, et al. Youth-onset Type 2 diabetes consensus report: current status, challenges, and priorities. Diabetes Care. 2016; 39:16351642. [PubMed: 27486237]

13. Zipf G, Chiappa M, Porter KS, Ostchega Y, Lewis BG, Dostal J. Vital Health Stat. Vol. 1. National Center for Health Statistics; 2013. National Health and Nutrition Examination Survey: plan and operations, 1999-2010.

14. Centers for Disease Control and Prevention (CDC); National Center for Health Statistics (NCHS). National Health and Nutrition Examination Survey Laboratory Protocol. Hyattsville, MD: US Department of Health and Human Services, Centers for Disease Control and Prevention; 2010.

15. Centers for Disease Control and Prevention (CDC); National Center for Health Statistics (NCHS). National Health and Nutrition Examination Survey Questionnaires. Hyattsville, MD: US Department of Health and Human Services, Centers for Disease Control and Prevention; 2010.

16. Centers for Disease Control and Prevention (CDC); National Center for Health Statistics (NCHS). Clinical Growth Charts. Hyattsville, MD: US Department of Health and Human Services, Centers for Disease Control and Prevention; 2000.

17. National High Blood Pressure Education Program Working Group. The fourth report on the diagnosis, evaluation, and treatment of high blood pressure in children and adolescents. Pediatrics. 2004; 114(2 Suppl):555-576. [PubMed: 15286277]

18. Expert panel on integrated guidelines for cardiovascular health and risk reduction in children and adolescents: summary report. Pediatrics. 2011; 128(Suppl 5):S213-256. [PubMed: 22084329]

19. Friedewald WT, Levy RI, Fredrickson DS. Estimation of the concentration of low-density lipoprotein cholesterol in plasma, without use of the preparative ultracentrifuge. Clin Chem. 1972; 18:499-502. [PubMed: 4337382]

20. KDIGO 2012 Clinical Practice Guideline for the Evaluation and Management of Chronic Kidney Diseases. Kidney Int Suppl. 2013; 3:1-150.

21. Weiss R, Santoro N, Giannini C, Galderisi A, Umano GR, Caprio S. Prediabetes in youth mechanisms and biomarkers. Lancet Child Adolesc Health. 2017; 1:240-248. [PubMed: 29075659]

22. Eklioglu BS, Atabek ME, Akyurek N, Alp H. Prediabetes and cardiovascular parameters in obese children and adolescents. J Clin Res Pediatr Endocrinol. 2016; 8:80-85. [PubMed: 26759114]

23. Li P, Jiang R, Li L, Wang Z, Li X, Liu C. Diagnostic performance of hemoglobin A $A_{1 c}$ for prediabetes and association with cardiometabolic risk factors in Chinese adolescents without diabetes. J Investig Med. 2012; 60:888-894.

24. Aradillas-Garcia C, Malacara JM, Garay-Sevilla ME, Guizar JM, Camacho N, De la CruzMendoza E, et al. Prediabetes in rural and urban children in 3 states in Mexico. J Cardiometab Syndr. 2007; 2:35-39. [PubMed: 17684445]

25. Whelton PK, Carey RM. The 2017 clinical practice guideline for high blood pressure. JAMA. 2017; 318:2073-2074. [PubMed: 29159375]

26. American Diabetes Association. Professional Practice Committee for the Standards of Medical Care in Diabetes-2016. Diabetes Care. 2016; 39(Suppl 1):S1-112. [PubMed: 26696671]

27. Nowicka P, Santoro N, Liu H, Lartaud D, Shaw MM, Goldberg R, et al. Utility of hemoglobin $\mathrm{A}(1 \mathrm{c})$ for diagnosing prediabetes and diabetes in obese children and adolescents. Diabetes Care. 2011; 34:1306-1311. [PubMed: 21515842]

28. Buse JB, Kaufman FR, Linder B, Hirst K, El Ghormli L, Willi S. Diabetes screening with hemoglobin $\mathrm{A}(1 \mathrm{c})$ versus fasting plasma glucose in a multiethnic middle-school cohort. Diabetes Care. 2013; 36:429-435. [PubMed: 23193207]

29. Standards of Medical Care in Diabetes-2017: Summary of Revisions. Diabetes Care. 2017; 40(Suppl 1):S4-S5. [PubMed: 27979887]

30. Bullard KM, Saydah SH, Imperatore G, Cowie CC, Gregg EW, Geiss LS, et al. Secular changes in US Prediabetes prevalence defined by hemoglobin $\mathrm{A}_{1 \mathrm{c}}$ and fasting plasma glucose: National Health and Nutrition Examination Surveys, 1999-2010. Diabetes Care. 2013; 36:2286-2293. [PubMed: 23603918] 


\section{What's new?}

- Adolescents with Type 2 diabetes are more likely to have cardiovascular disease (CVD) risk factors but there are few data available among adolescents with prediabetes.

- $\quad$ Using a nationally representative survey, we found that US adolescents with prediabetes were more likely to have obesity, low HDL-cholesterol, high triglycerides and elevated liver transaminase than adolescents with normal glucose.

- We observed increasing odds of these health outcomes beginning at levels below the cut-off point for prediabetes.

- Addressing prediabetes and comorbidity in youth is important to reduce the risk of Type 2 diabetes and the risk of substantial long-term health consequences. 
Percent with Prediabetes

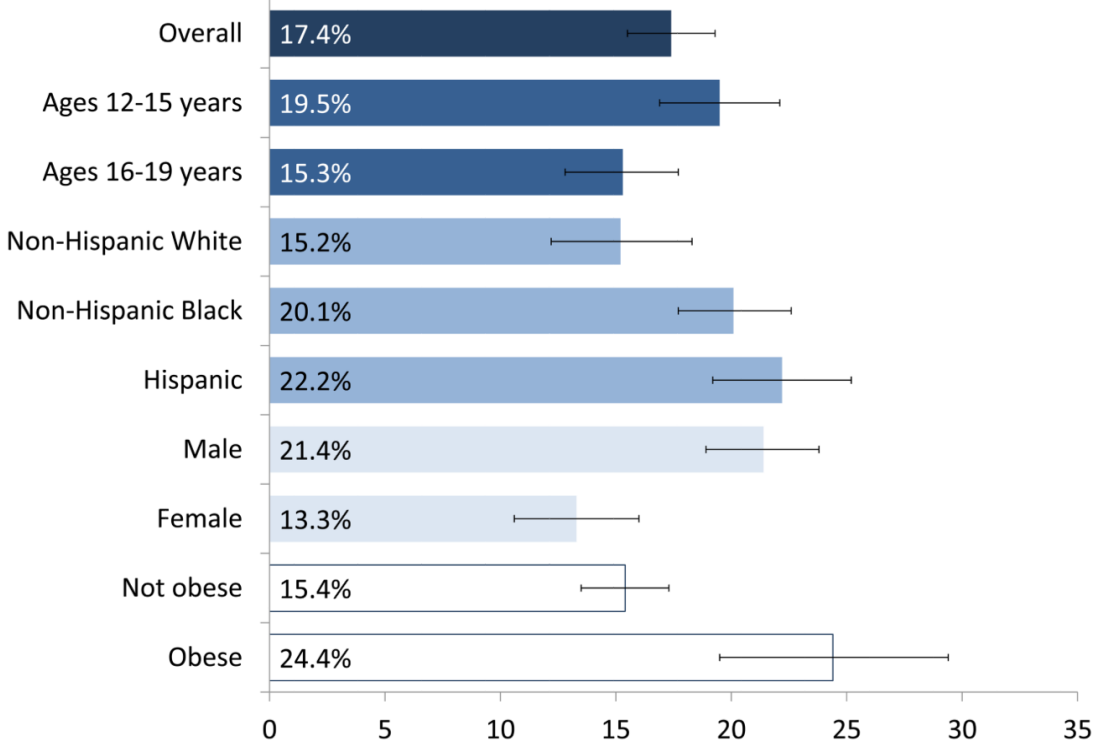

Figure 1.

Weighted per cent with prediabetes overall and stratified by participant characteristics, 2005-2014 National Health and Nutrition Examination Survey. 

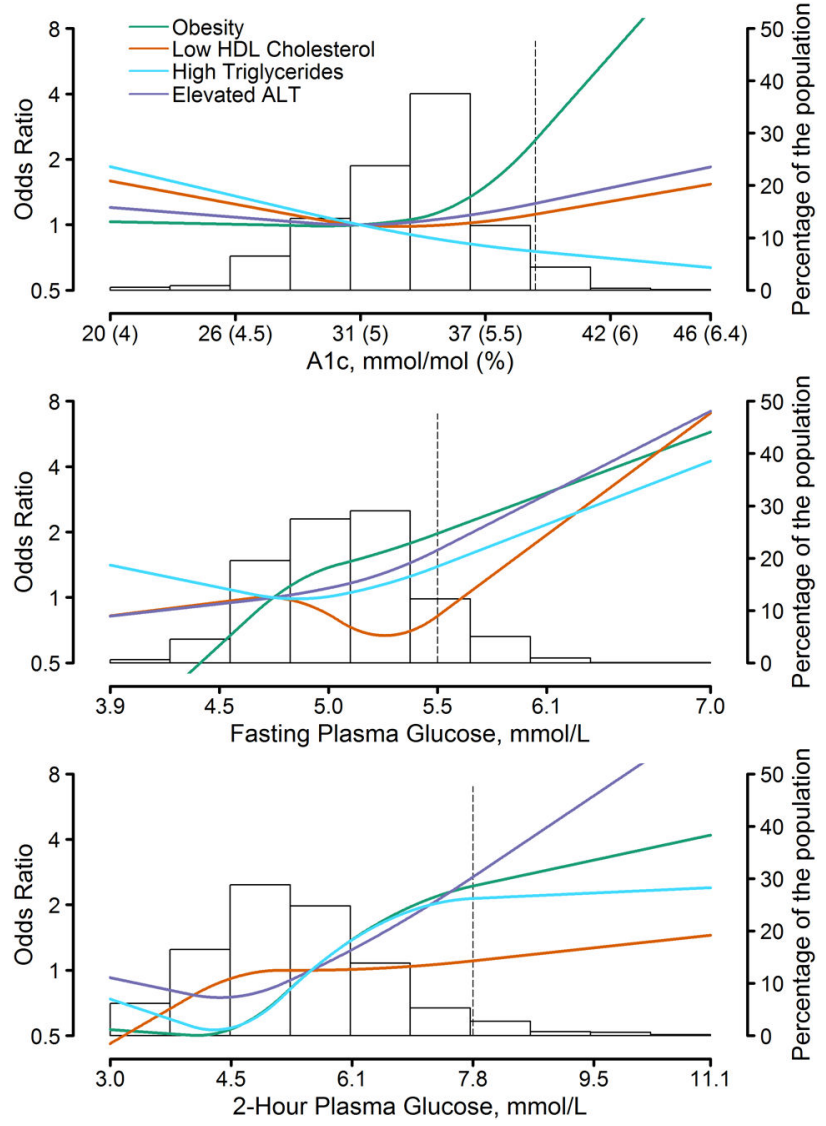

Figure 2.

Adjusted relative odds of obesity, low HDL-cholesterol, high triglycerides and elevated ALT by $\mathrm{HbA}_{1 \mathrm{c}}$ (upper), fasting plasma glucose (middle) and 2-h plasma glucose (lower). Background histogram of the glucose marker is displayed on the right-hand axis. The prediabetes cut-off point for each marker is indicated by a dashed line. ALT, alanine aminotransferase; HDL, high-density lipoprotein. 2005-2014 National Health and Nutrition Examination Survey. 
Table 1

Participant characteristics (se) by diabetes status, 2005-2014 National Health and Nutrition Examination Survey*

\begin{tabular}{|c|c|c|c|}
\hline & Normal glucose $N=2032$ & Prediabetes $^{\dagger} \mathbf{N}=811$ & P-value t $^{t}$ \\
\hline Age, years & $15.5(0.06)$ & $15.3(0.13)$ & 0.08 \\
\hline Race/ethnicity $\xi$ & & & 0.02 \\
\hline Non-Hispanic white, $\%$ & $60.3(2.25)$ & $51.4(3.71)$ & \\
\hline Non-Hispanic Black, \% & $14.3(1.26)$ & $17.1(1.87)$ & \\
\hline Hispanic, $\%$ & $18.0(1.50)$ & $24.5(2.61)$ & \\
\hline Female, $\%$ & $51.8(1.77)$ & $37.8(2.58)$ & $<0.001$ \\
\hline Household income $<\$ 20000, \%$ & $14.9(1.43)$ & $16.8(1.95)$ & 0.37 \\
\hline Smoke, $\%$ & $14.6(1.15)$ & $16.0(2.37)$ & 0.61 \\
\hline Total daily calories, kcal & $2115(27.7)$ & $2149(65.1)$ & 0.59 \\
\hline Per cent of calories from saturated fat, mean $\%$ & $11.1(0.11)$ & $11.4(0.21)$ & 0.18 \\
\hline Obese, $\%$ & $18.4(1.33)$ & $28.6(2.68)$ & $<0.001$ \\
\hline
\end{tabular}


Table 2

Adjusted percentage (95\% CI) and odds ratios (95\% CI) of obesity, hypertension, high total cholesterol, high LDL-cholesterol, low HDL-cholesterol, high triglycerides, elevated ALT and albuminuria associated with prediabetes status, 2005-2014 National Health and Nutrition Examination Survey

\begin{tabular}{|c|c|c|c|c|}
\hline & \multicolumn{3}{|c|}{ Percentages } & \multirow{2}{*}{ Odds ratios ${ }^{t}$} \\
\hline & Normal glucose & Prediabetes & P-value & \\
\hline \multicolumn{5}{|l|}{ Obesity } \\
\hline Unadjusted & $18.4(15.9-21.2)$ & $28.6(23.6-34.3)$ & $<0.001$ & $1.78(1.32-2.38)$ \\
\hline Multivariable model $1 *$ & $18.1(15.6-20.9)$ & $28.4(23.1-34.4)$ & $<0.001$ & $1.79(1.33-2.41)$ \\
\hline Multivariable model $2^{\dagger}$ & $17.7(15.1-20.7)$ & $28.6(22.9-35.0)$ & $<0.001$ & $1.86(1.35-2.55)$ \\
\hline \multicolumn{5}{|l|}{ Hypertension } \\
\hline Unadjusted & $1.8(1.2-2.9)$ & $4.3(2.6-6.9)$ & 0.01 & $2.36(1.24-4.52)$ \\
\hline Multivariable model $1 *$ & $1.6(1.0-2.7)$ & $3.2(1.8-5.6)$ & 0.03 & $2.04(1.06-3.93)$ \\
\hline Multivariable model $2^{\dagger}$ & $1.2(0.7-2.1)$ & $2.1(1.1-4.0)$ & 0.11 & $1.77(0.88-3.54)$ \\
\hline \multicolumn{5}{|l|}{ High total cholesterol } \\
\hline Unadjusted & $8.0(6.5-9.8)$ & $10.7(7.7-14.9)$ & 0.15 & $1.38(0.89-2.14)$ \\
\hline Multivariable model $1 *$ & $7.9(6.4-9.6)$ & $11.0(7.8-15.3)$ & 0.11 & $1.45(0.92-2.27)$ \\
\hline Multivariable model $2^{\dagger}$ & $7.7(6.1-9.5)$ & $9.7(6.9-13.6)$ & 0.26 & $1.30(0.82-2.06)$ \\
\hline \multicolumn{5}{|l|}{ High LDL-cholesterol } \\
\hline Unadjusted & $5.9(4.6-7.6)$ & $9.4(6.0-14.4)$ & 0.09 & $1.65(0.93-2.92)$ \\
\hline Multivariable model $1 *$ & $5.7(4.4-7.3)$ & $9.3(5.9-14.4)$ & 0.07 & $1.71(0.95-3.10)$ \\
\hline Multivariable model $2^{\dagger}$ & $5.4(4.0-7.2)$ & $8.3(5.3-12.6)$ & 0.12 & $1.59(0.88-2.88)$ \\
\hline \multicolumn{5}{|l|}{ Low HDL-cholesterol } \\
\hline Unadjusted & $10.6(8.7-12.7)$ & $19.4(15.6-23.9)$ & $<0.001$ & $2.04(1.43-2.91)$ \\
\hline Multivariable model $1 *$ & $9.9(8.2-11.8)$ & $17.9(13.8-23.0)$ & $<0.001$ & $2.00(1.36-2.93)$ \\
\hline Multivariable model $2^{\dagger}$ & $7.8(6.5-9.5)$ & $12.1(8.8-16.5)$ & 0.02 & $1.62(1.08-2.44)$ \\
\hline \multicolumn{5}{|l|}{ High triglycerides } \\
\hline Unadjusted & $10.8(9.1-12.8)$ & $18.4(14.4-23.3)$ & $<0.001$ & $1.87(1.35-2.58)$ \\
\hline Multivariable model $1 *$ & $9.9(8.4-11.7)$ & $17.3(13.2-22.3)$ & $<0.001$ & $1.90(1.35-2.68)$ \\
\hline Multivariable model $2^{\dagger}$ & $8.9(7.3-10.8)$ & $13.6(9.7-18.6)$ & 0.01 & $1.61(1.12-2.30)$ \\
\hline \multicolumn{5}{|l|}{ Elevated ALT } \\
\hline Unadjusted & $5.1(3.9-6.5)$ & $12.7(9.5-16.6)$ & $<0.001$ & $2.73(1.70-4.38)$ \\
\hline Multivariable model $1 *$ & $4.2(3.3-5.4)$ & $10.8(7.7-15.1)$ & $<0.001$ & $2.74(1.73-4.35)$ \\
\hline Multivariable model $2^{\dagger}$ & $2.9(2.1-4.0)$ & $5.9(3.6-9.4)$ & 0.01 & $2.09(1.19-3.67)$ \\
\hline \multicolumn{5}{|l|}{ Albuminuria } \\
\hline Unadjusted & $11.8(10.0-13.9)$ & $11.6(8.6-15.6)$ & 0.95 & $0.99(0.66-1.48)$ \\
\hline Multivariable model $1 *$ & $10.4(8.6-12.5)$ & $11.5(8.2-15.9)$ & 0.61 & $1.12(0.72-1.72)$ \\
\hline Multivariable model $2^{\dagger}$ & $9.6(7.6-12.0)$ & $11.6(7.6-17.2)$ & 0.39 & $1.24(0.76-2.02)$ \\
\hline
\end{tabular}


CI, confidence interval; ALT, alanine aminotransferase; CVD, cardiovascular disease.

* Multivariable model 1 adjusted for age, race/ethnicity and sex.

${ }^{\dagger}$ Multivariable model 2 adjusted for age, race/ethnicity, sex, income, smoking, daily calories consumed, per cent of calories from saturated fat, BMI (except obesity model) and systolic BP (albuminuria model only).

Fodds ratio determined from logistic regression. 